\title{
Diagnostic imaging of a solid pseudopapillary tumour of the pancreas in a 20-year-old woman - a case study
}

\author{
Katarzyna Z. Rajtar ${ }^{1}$, Katarzyna Sznajder ${ }^{1}$, Katarzyna M. Milto ${ }^{2}$ \\ ${ }^{1}$ Department of Imaging Diagnostics, Regional Medical Centre, Opole, Poland \\ ${ }^{2}$ St John's Hospital, Edinburgh, United Kingdom
}

Gastroenterology Rev 2016; 11 (3): 214-217

DOI: $10.5114 / p g .2016 .57882$

\begin{abstract}
Address for correspondence: Katarzyna Z. Rajtar MD, PhD, Department of Imaging Diagnostics, Regional Medical Centre, al. W. Witosa 26, 45-418 Opole, Poland, phone: +48 7284057 59, e-mail: rajtar.k@gmail.com
\end{abstract}

Solid pseudopapillary neoplasm (SPN) of the pancreas is a rare tumour of uncertain histopathological origin derived from the exocrine pancreas and most often first detected on radiological imaging. It predominantly affects young women in their second and third decades of life. Solid pseudopapillary neoplasm has a low-grade malignant potential with excellent post-surgical curative rates. The lesions can be detected and differentiated from other pancreatic lesions by ultrasound imaging (US), computed tomography (CT), and magnetic resonance imaging (MRI). We report a case of solid pseudopapillary neoplasm of the pancreas that was diagnosed on CT imagining and confirmed on postoperative histological examination.

A 20-year-old woman with no prior medical history was admitted to the Emergency Department with a 2-day history of nausea, vomiting, and diarrhoea. She described a one-year history of abdominal 'fullness' but no other symptoms of note. Her vital observations were stable. On physical examination there was a palpable mass in the right hypochondriac region. Haematological and biochemical tests, including tumour markers (CEA, CA-125, CA19-9, hCD, $\alpha$-fetoprotein), were within normal limits. Transabdominal ultrasound scan revealed a well-circumscribed, heterogeneous lesion with anechoic areas. It measured $80 \times 70 \mathrm{~mm}$ and was situated between the right kidney and the right lobe of the liver. A multiphase contrast-enhanced computer tomography scan was ordered. It showed a round, retroperitoneal lesion with cystic and solid components measuring $70 \times 61 \times 96 \mathrm{~mm}$ located in the head of pancreas. The mass was separated from its surroundings by a wall of variable thickness (Figures $1 \mathrm{~A}, \mathrm{~B}$ ). There were some linear areas of calcification on the rim and in the in- ternal content as well as cystic areas filled with fluid of 30 Hounsfield unit (HU) mean density (Figure 2). Its upper margin contained multiple areas of soft-tissue density that enhanced with contrast (Figure 3). The lesion exerted a mass effect on the visceral surface of the right liver lobe, the head of the pancreas, and the duodenum, resulting in displacement of these structures. The remaining intra-abdominal organs were of normal radiological appearance with no lymphadenopathy. The above findings were suggestive of a Frantz tumour of the pancreas. Gastroscopy revealed inflammation of the gastric antrum but no other changes. A chest radiograph was unremarkable.

The patient was transferred to the Surgical Oncology Department and was assessed for surgical intervention. She had undergone a radical excision of the mass by an open approach with an upper midline incision. On gross pathological examination there was a cystic, multiloculated mass measuring $90 \times 75 \times 65 \mathrm{~mm}$ and surrounded by a stiff, focally calcified wall. The largest cyst measured $60 \mathrm{~mm}$ and was filled with dark brown 'chocolate' fluid. The mass was fixed to the anterolateral part of the head of the pancreas. The histopathological examination revealed pancreatic pseudopapillary neoplasm positive for cytokeratin (CK) 8/18, vimentin, ER, and PR and equivocal for synaptophysin. There was no involvement of the incisional margins. The patient has made an uneventful post-operative recovery. Follow-up transabdominal ultrasound examinations were performed every month for the first half a year and every 3 months thereafter. Abdominal CT scan was repeated every 6 months for 1 year. There were no signs of tumour recurrence in any of the follow up scans. The patient 

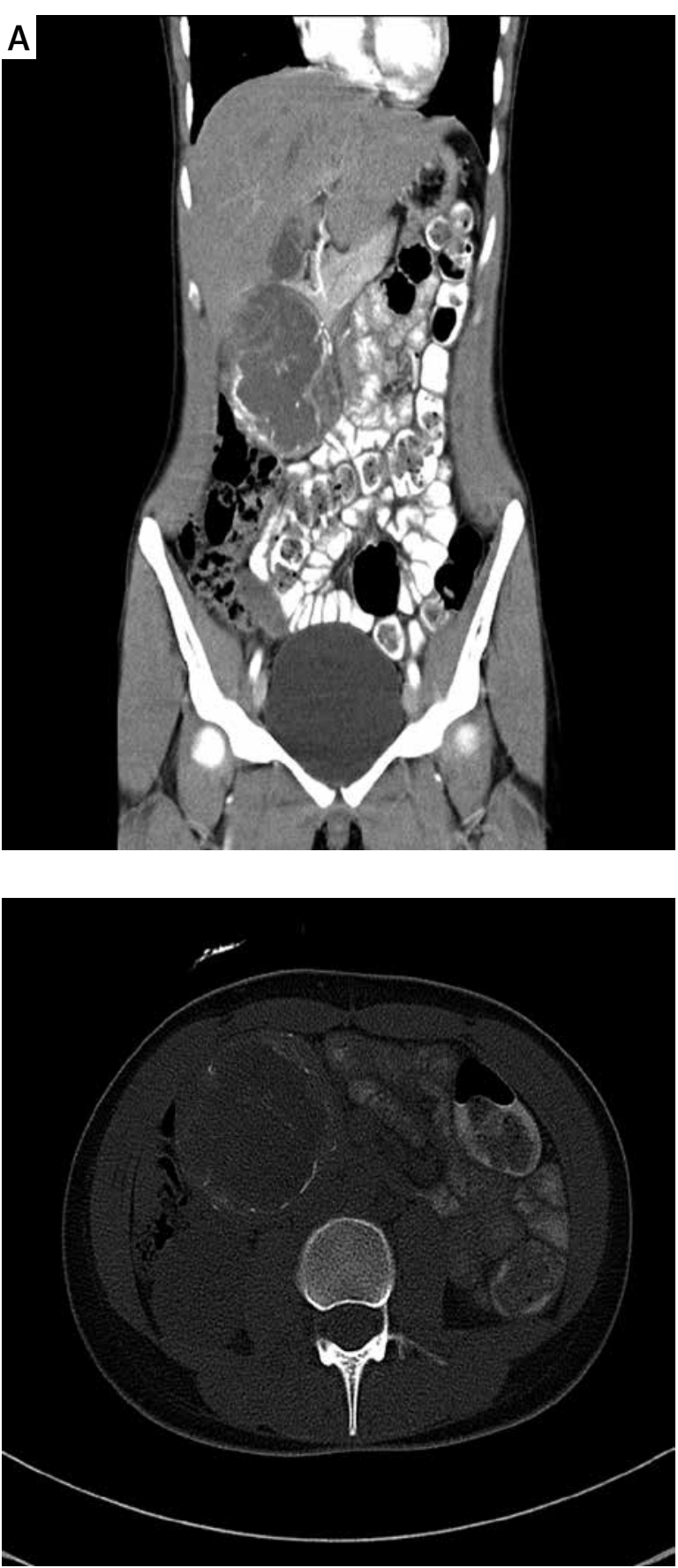

Figure 2. Linear areas of calcification on the rim and within the lesion, as illustrated on bone window CT scan

remained asymptomatic and did not require pharmacological therapy or any further surgical intervention. The planned follow-up includes abdominal ultrasound scan every half a year.

Solid pseudopapillary tumour (SPT) is a rare exocrine pancreatic tumour, accounting for $1-2 \%$ of all tumours of the pancreas [1]. The first 2 cases were encountered

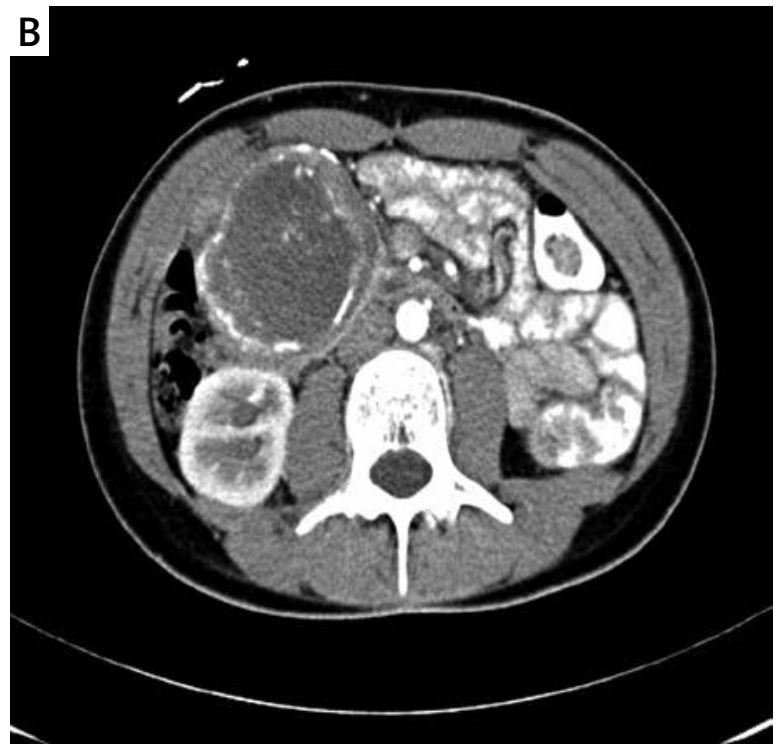

Figure $1 \mathrm{~A}, \mathrm{~B}$. Arterial phase of abdominal CT scan revealing capsulated pancreatic mass with cystic and solid components

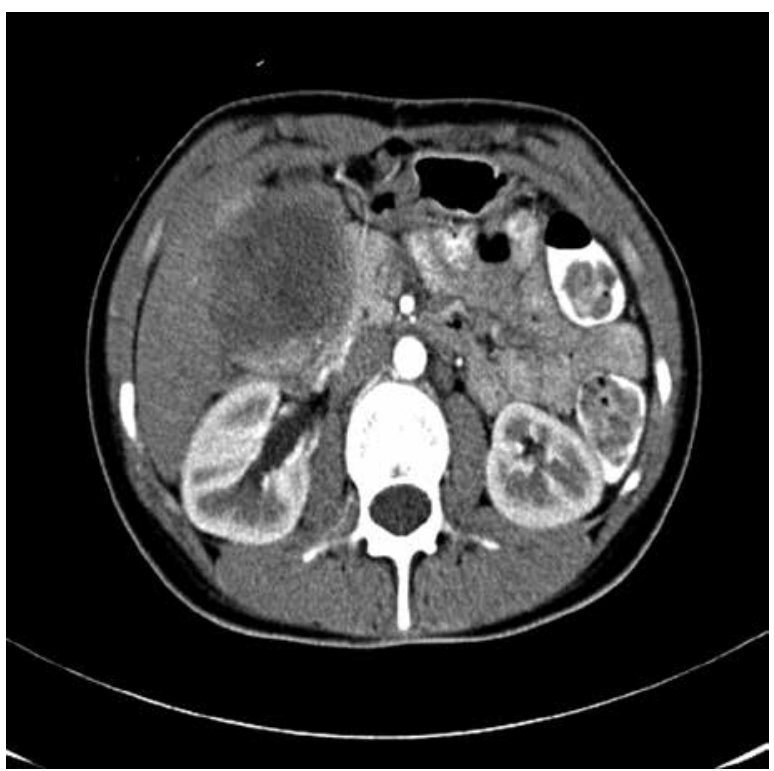

Figure 3. Soft-tissue part of the lesion shows similar enhancement to pancreatic parenchyma on venous phase of CT

and described by Virginia Frantz in 1959. The male to female ratio is $1: 10$, and the mean age at presentation is 22 years [2]. There are only occasional case reports of SPT of the pancreas in children or men [2]. The origin and pathogenesis of SPT remain unclear. Based on the immunohistological profile, many researchers favour the theory that SPT originates from multipotent stem cells. 
Other authors, however, suggest that female sex hormones may play a role in tumorigenesis, but a definitive causal relationship has not been proven $[3,4]$.

The clinical presentation of this tumour is usually nonspecific. Abdominal discomfort or vague, generalised pain are the most common symptoms. As the lesions enlarge, they may compress the neighbouring structures and exert a mass effect, resulting in symptoms such as nausea, vomiting, or jaundice. The largest case series to date by Papavramidis and Papavramidis [2] comprised 718 cases of SPT and revealed that only $1 \%$ of patients with pancreatic head tumours were jaundiced at presentation. Rarely, the patient may present acutely with haemoperitoneum caused by the rupture of tumour capsule, pancreatic duct obstruction, or ischaemia. However, one third of SPTs are asymptomatic and are found incidentally on routine physical examination or on imaging studies. There are no pathognomonic features on blood testing to aid the diagnosis. Tumour markers are usually within normal levels. Because of the low-grade malignant potential and good prognosis after complete resection of the tumour, the correct diagnosis is crucial prior to invasive intervention.

Trans-abdominal ultrasound and cross-sectional abdominal CT scan are usually sufficient to identify the tumour [5]. Magnetic resonance imaging scanning, endoscopic ultrasound fine-needle aspiration (EUS-FNA), trans-abdominal fine-needle aspiration biopsy, or magnetic resonance cholangiopancreatography (MRCP) are the second-line investigations that may also assist a preoperative diagnosis of SPT.

Radiological imaging typically demonstrates an encapsulated mass and occasional foci of dystrophic calcification that, depending on their size, are easily visible in about $30 \%$ of cases on CT and less commonly on US or MRI examinations. Non-encapsulated lesions have also been reported, but they were probably detected at an early stage, before formation of the capsule [6]. Frequently, an ultrasound scan demonstrates a homogenous or heterogenous well-encapsulated mass composed of solid echogenic and hypoechogenic components, with calcification and possible displacement of nearby structures. Computed tomography cross-sectional imaging revealed an encapsulated, well-defined mass with central areas of calcification, necrosis, haemorrhage, and cystic degeneration. The peripheral enhancement, characterised by a similar Hounsfield unit density as the nearby pancreatic parenchyma, is usually present in both arterial and venous phases of the CT scan. This feature differentiates SPT from adenocarcinomas, which are usually hypo-attenuated on the venous phase and from pancreatic neuroendocrine tumours
(pNET) that enhance on the arterial phase [5]. Some authorities also advocate the additional use of magnetic resonance imaging because of its ability to delineate tissue characteristics such as haemorrhage and necrosis [7]. In keeping with the definition, SPNs are usually solid, but cavities filled with necrotic masses or blood resulting from acute haemorrhages can often be noted in large lesions. The apparent diffusion coefficient (ADC) value, which depends on the degree of restriction in water diffusion and tissue cellularity, is usually higher in SPTs than in other malignant tumours [6].

The pathological diagnosis of SPT is based mainly on well-defined solid and cystic structure and characteristic pseudopapillary features under the microscope. On gross examination, the tumour tissue is usually well demarcated from the normal pancreas by a fibrous capsule. The microscopic features of SPT include solid areas that alternate with a pseudopapillary pattern composed of a fibrovascular stalk surrounded by several layers of epithelial cells. Immunohistochemical staining is frequently performed to confirm the diagnosis. Solid pseudopapillary tumour is typically positive for vimentin and antitrypsin and negative for trypsin and chymotrypsin [4].

The differential diagnosis for such tumours includes acinar cell carcinoma, pancreatoblastoma, neuroendocrine neoplasms, metastatic adenocarcinomas and non-neoplastic lesions such as post-inflammatory pseudocyst, parasitic cyst, or ectopic spleen [2]. As the pseudo-papillary pattern can resemble trabecular architecture, SPT may be mistakenly recognised as a non-functional pancreatic neuroendocrine tumour in adults [4]. The differential diagnosis in affected children is limited to pancreatoblastoma, a more aggressive tumour with equal distribution among young boys and girls.

Surgical removal of the tumour is the treatment of choice, but the extent of resection is still debated. In a retrospective series, Li et al. [8] compared 'minimised' and 'standard' pancreatic resections of SPTs. Both groups had similar long-term survival and morbidity rates, but patients subjected to 'standard resections' had higher transfusion rates, longer operating times, and longer hospitalisation periods. On the other hand, the mean follow-up period was 29 months, which is insufficient to demonstrate a difference in long-term survival. Therefore, conservative resection with preservation of as much pancreatic tissue as possible is still regarded as the treatment of choice [5]. The overall 5-year survival rate of patients with SPN is about 95\% [2].

Solid pseudopapillary tumour is a rare pancreatic tumour with good prognosis after complete resection. While clinical signs and symptoms are relatively nonspecific, characteristic findings on imaging and histolo- 
gy separate the tumour from more malignant pancreatic tumours. In the reported patient, abdominal CT scans were sufficient to make the correct diagnosis and to aid curative surgical treatment.

\section{Conflict of interest}

The authors declare no conflict of interest.

\section{References}

1. Colman KM, Doherty MC, Bigler SA. Solid pseudopapillary tumor of the pancreas. Radiographic 2003; 23: 1644-8.

2. Papavramidis T, Papavramidis S. Solid pseudopapillary tumors of pancreas: review of 718 patients reported in English literature. J Am Coll Surg 2005; 200: 965-72.

3. Shuja A, Alkimaw KA. Solid pseudopapillary tumor: a rare neoplasm of the pancreas. Gastroenterol Rep 2014; 2: 145-9.

4. Zhang H, Liang TB, Wang WL, et al. Diagnosis and treatment of solid pseudopapillary tumor of the pancreas. Hepatobil Pancreat Dis Int 2006; 5: 454-8.

5. Dan D, Rambally R, Cawich S, et al. Solid pseudopapillary neoplasms of the pancreas: a report of two cases. Hindawi Publishing Corporation. Case Rep Med 2014; 2014: 356-79.

6. Burdan F, Mocarska A, Guz E, et al. Solid-pseudopapillary neoplasm of the pancreas - comparisons between magnetic resonance and histological findings. Pol J Pathol 2013; 64: 216-23.

7. Procacci C, Graziani R, Bicego E, et al. Papillary cystic neoplasm of the pancreas: radiological findings. Abdom Imaging 1996; 21: 554-8.

8. Li Z, Zhang Z, Liu X, et al. Solid pseudopapillary tumor of the pancreas: the surgical procedures. Surgery Today 2011; 41: 91-6.

Received: 3.04.2015

Accepted: 10.07.2015 